\title{
Desenvolvimento baseado em testes com aplicação de critérios de teste no ensino introdutório de computação
}

\author{
Yuri Rafael Grajefe Feitosa \\ Universidade Tecnológica Federal do \\ Paraná, Cornélio Procópio, PR, Brasil \\ yurirafael@alunos.utfpr.edu.br
}

\author{
Marco Aurélio Graciotto Silva \\ Universidade Tecnológica Federal do \\ Paraná, Campo Mourão, PR, Brasil \\ magsilva@utfpr.edu.br
}

\author{
José Augusto Fabri \\ Universidade Tecnológica Federal do \\ Paraná, Cornélio Procópio, PR, Brasil \\ fabri@utfpr.edu.br
}

Ensinar os alunos a programar é um processo complexo [7]. De modo geral, existe uma linha de desenvolvimento que deve ser seguida, começando pela lógica, depois a lógica de programação e por fim os algoritmos [3]. Dentre as abordagens para ensino de programação [9], o teste de software pode ser considerado para tratar a resolução de problemas [4]. Assim, observam-se indícios de que o teste de software criterioso pode contribuir no processo de aprendizagem e desenvolvimento de código [11].

Um caso de teste consiste em um conjunto de entradas, condições de execução e saída esperada para um programa [5]. A reflexão sobre a elaboração de um caso de teste na fase introdutória do ensino de programação pode induzir o aluno a pensar melhor na resolução de um determinado problema computacional e, consequentemente, chegar a uma resposta de forma eficiente, com menos tentativas e erros no percurso [2]. Em estudo correlato, observou-se que o uso de critérios de teste em conjunto com o aprendizado dirigido a teste contribuem para a melhoria da qualidade dos programas e dos casos de teste [8]. No entanto, naquele trabalho foi considerado um conjunto limitado de critérios de teste e não foi considerada a relação entre casos de teste, requisitos de teste e objetivos de aprendizagem associados a cada atividade de aprendizagem.

O objetivo desta pesquisa consiste em estabelecer uma abordagem de desenvolvimento orientado a testes (TDD - Test-Driven Development) que empregue critérios de teste de forma escalonada e associada a objetivos de atividades de aprendizagem de programação. Desta forma, busca-se aperfeiçoar o desenvolvimento da lógica computacional dos alunos nos primeiros degraus de aprendizagem de programação nos cursos de computação e áreas afins.

A abordagem proposta consiste em conciliar os critérios de teste de software ao TDD na elaboração da lógica computacional do problema proposto com a finalidade de reduzir a curva de aprendizado do aluno. TDD é uma técnica iterativa de design de software associada a teste de software organizada em três etapas: definição dos casos de teste, implementação do código e refatoração [1]. Um critério de teste permite avaliar o software quanto a um determinado tipo de erro, podendo ser associado a objetivos de atividades de aprendizagem. Desta forma, critérios de teste de software podem ser utilizados para orientar o aluno a pensar em resolver problema de maneira eficiente, evitando abordagens de tentativa e erro.

Fica permitido ao(s) autor(es) ou a terceiros a reprodução ou distribuição, em parte ou no todo, do material extraído dessa obra, de forma verbatim, adaptada ou remixada, bem como a criação ou produção a partir do conteúdo dessa obra, para fins não comerciais, desde que sejam atribuídos os devidos créditos à criação original, sob os termos da licença CC BY-NC 4.0.

EduComp'21, Abril 27-30, 2021, Jataí, Goiás, Brasil (On-line)

(c) 2021 Copyright mantido pelo(s) autor(es). Direitos de publicação licenciados à Sociedade Brasileira de Computação (SBC).
Como exemplo, considere o cenário de alunos ingressante dos cursos de computação (CS1), submetidos a uma série de exercícios, na linguagem $\mathrm{C}$, com a finalidade de avaliar o método proposto. Essas atividades têm o nível de dificuldade escalonado, começando com a soma de dois números, depois um problema que envolva estrutura de decisão, após isso, decisão e repetição, assim por diante. Tais atividades são avaliadas de forma automática por juízes online, considerando conjuntos de casos de teste e implementação de referência, ambos definidos pelo instrutor, que satisfazem determinados critérios e que são pertinentes aos objetivos de aprendizagem.

Para cada exercício, o aluno deve criar casos de teste antes da implementação do programa. Como é uma disciplina introdutória à programação, não seria razoável que definisse casos de teste automatizados. Como alternativa, cada caso de teste deve ser especificado de forma simplificada, informando-se apenas os dados de entrada e o resultado esperado como parte da documentação $[6,10]$. Cada caso de teste deve ser processado pela ferramenta associada à abordagem proposta, fornecendo os dados de entrada como parâmetros da função ou do programa e comparando o resultado obtido com o resultado esperado. Os resultados podem ser obtidos tanto em relação ao programa do aluno quanto à solução de referência.

Em um primeiro momento, é esperado que o caso de teste falhe em relação ao código do aluno (dada a sua inexistência). Neste instante, ele prossegue para a implementação de sua solução até que o caso de teste passe. A execução do caso de teste com relação à solução de referência deve ser utilizada para discernir um erro de programação de um erro de compreensão do problema, o que deve ser informado após repetidas tentativas sem perceber o equívoco. Quando a solução do aluno satisfizer os casos de teste criados, os resultados de falha do teste da solução dele com a solução de referência devem ser considerados para guiá-lo a criar novos casos de teste, explorando requisitos de teste e problemas típicos relacionados aos conceitos sendo aprendidos.

Como forma de verificação da eficácia da abordagem proposta, será feita a comparação com a abordagem tradicional de ensino, considerando instrumentos de avaliação como FCS1 [12].

Ao considerar os critérios de teste para auxiliar a construção de programas, a presente proposta busca induzir a melhora na aprendizagem dos alunos, principalmente no desenvolvimento da lógica computacional. Portanto, espera-se que os alunos pensem mais antes de resolver um problema e que consequentemente a solução desse problema seja feita da melhor forma possível.

\section{REFERÊNCIAS}

[1] Kent Beck. 2002. Test-Driven Development: By Example (1 ed.). Addison-Wesley Professional, EUA. 240 pages.

[2] Stephen H. Edwards. 2004. Using software testing to move students from trial-anderror to reflection-in-action. In 35th SIGCSE Technical Symposium on Computer 
Science Education (35 ed.) (Norfolk, VA, EUA). ACM, New York, NY, EUA, 26-30. https://doi.org/10.1145/971300.971312

[3] André Luiz Villar Forbellone and Henri Frederico Eberspächer. 2005. (3 ed.) Pearson, São Paulo, SP, Brasil. 218 pages.

[4] Pei Hsia and Frederick E. Petry. 1980. A Framework for Discipline in Programming. Transactions on Software Engineering SE-6, 2 (March 1980), 226-232. https://doi.org/10.1109/TSE.1980.234479

[5] ISO, IEC, and IEEE. 2017. ISO/IEC/IEEE 24765:2017 - Systems and software engineering - Vocabulary. Padrão Internacional. , 525 pages. https://doi.org/10. 1109/IEEESTD.2017.8016712

[6] Vesa Lappalainen, Jonne Itkonen, Ville Isomöttönen, and Sami Kollanus. 2010 ComTest: a tool to impart TDD and unit testing to introductory level programming. In 15th Annual Conference on Innovation and Technology in Computer Science Education (15 ed.) (Bilkent, Ankara, Turquia). ACM, New York, NY, EUA, 63-67. https://doi.org/10.1145/1822090.1822110

[7] Andrew Luxton-Reilly, Simon, Ibrahim Albluwi, Brett A. Becker, Michail Giannakos, Amruth N. Kumar, Linda Ott, James Paterson, Michael James Scott, Judy Sheard, and Claudia Szabo. 2018. Introductory Programming: A Systematic Literature Review. In Proceedings of the 23rd Annual ACM Conference on Innovation and Technology in Computer Science Education (23 ed.) (Larnaca, Cyprus). ACM, New York, NY, EUA, 55-106. https://doi.org/10.1145/3293881.3295779
[8] Bruno Henrique Pachulski Camara and Marco Aurélio Graciotto Silva. 2016. A Strategy to Combine Test-Driven Development and Test Criteria to Improve Learning of Programming Skills. In 47th ACM Technical Symposium on Computing Science Education (47 ed.) (Memphis, TN, EUA). ACM, New York, NY, EUA, 443448. https://doi.org/10.1145/2839509.2844633

[9] Arnold Pears, Stephen Seidman, Lauri Malmi, Linda Mannila, Elizabeth Adams, Jens Bennedsen, Marie Devlin, and James Paterson. 2007. A Survey of Literature on the Teaching of Introductory Programming. In Working Group Reports on ITiCSE on Innovation and Technology in Computer Science Education (ITiCSEWGR 2007) (Dundee, Scotland). ACM, New York, NY, EUA, 204-223. https: //doi.org/10.1145/1345443.1345441

[10] Python Software Foundation. 1999. doctest - Test interactive Python examples. Software. https://docs.python.org/3/library/doctest.html

[11] Lilian Passos Scatalon, Jeffrey C. Carver, Rogério Eduardo Garcia, and Ellen Francine Barbosa. 2019. Software Testing in Introductory Programming Courses: A Systematic Mapping Study. In Proceedings of the 50th ACM Technical Symposium on Computer Science Education (50 ed.) (Minneapolis, MN, EUA). ACM, New York, NY, EUA, 421-427. https://doi.org/10.1145/3287324.3287384

[12] Allison Elliott Tew and Mark Guzdial. 2011. The FCS1: A Language Independent Assessment of CS1 Knowledge. In 42nd ACM Technical Symposium on Computer Science Education (42 ed.) (Dallas, TX, EUA). ACM, New York, NY, EUA, 111-116. https://doi.org/10.1145/1953163.1953200 